\title{
The M otor Drive Part Hall Memory Function Detection
}

\author{
Yu Bao-jun ${ }^{1, a}$, XinTian-tian ${ }^{2, b}$ Lin Jie-qiong $^{3, c^{*}}$ and Tian Peng ${ }^{4, d}$ \\ Changchun University of Technology, China \\ abruce5257@126.com; bchutian200172@163.com; 'linjieqiong@ccut.edu.cn; ${ }^{d}$ 82316614@qq.com
}

Keyword: Hall memory function, Motor parts; Digital filter, Square wave parameters detection

\begin{abstract}
In modern vehicles, part of the DC motor driven components use DC motor with hall of memory function, it is using the square wave produced by the Hall sensor as dc motor position feedback signal of the mechanical parts, these parts should pass position memory test to determine whether qualified after assembled. This paper use a Hall sensor signal data acquisition system based on virtual instrument software, when the DC motor is rotating, using the AD card collect the voltage square wave signals produced by the Hall sensor, and using graphical programming language for digital filtering and data analysis processing to get the signal parameters such as the duty-cycle and so on, and according to the given parameters to judge. Experiments show that compared to the full-scale square wave counting method, the parameter test of the system has the advantages of high precision, fast response characteristics.
\end{abstract}

\section{Introduction}

Now, In the car motor driving parts, parts are required to memory those position, take the electric seat basin as example, the cushion is generally driven by $3 \mathrm{dc}$ motors, respectively control the seat height, seat angle and anteroposterior position, the cushion with memory function is mainly based on the parameter information of the motor as the position detection parameters, such as the number of square wave issued Hall sensor motor driven by electrical appliances square wave, the quality of the motor or current pulse number Hall is more common, while Hall memory function of the current detection methods for the common counting method, which make the component from running one cycle, collecting the number of square wave that produced by Hall sensor installed in the motor during the process of moving parts, and compared to the number of known range to determine which parts of Hall memory function are qualified.

With the continuous development of industrial technology, the measurement of waveform parameters is also growing, dynamic change and precision of parameters has important requirements. When the motor spindle rotates, if according to the number of square wave to judge whether the motor has the memory function, it will turn a few laps to reflect, and not timely alarm, bring about unnecessary waste of time, this paper will detect square wave's parameters that produced by the seat motor spindle rotate a circle, in order to obtain the key parameters of experimental data, establishing a test system based on AD card of IPC, and using digital filtering techniques, the square wave's parameter detection method for rapid judgment.

\section{The Hall Memory Function And The Composition And Control Of The Signal Acquisition Platform}

The system consists of three parts, they are the mechanical parts, pneumatic components and electrical detection of control components. In the detection process, the measured parts should be clamp by fixture, using high power adjustable dc power supply to the motor drive unit of dc motor, the system uses M series AD PCI6221 produced by American NI company to acquire square wave voltage signal from the Hall sensor. Figure 1 shows the block diagram of the signal acquisition system. 


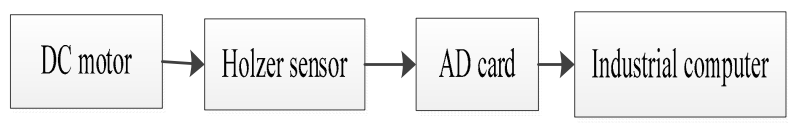

Figure 1 block diagram of the signal acquisition system

Hall sensor is a kind of magnetic sensor that is based on Hall effect ${ }^{[1]}$, when after alternating magnetic field will produce an output voltage pulse, it has the characteristics of small size, good dynamic performance, high sensitivity and strong anti-interference ability, it plays an important role in locomotive control system.

According to the counting of square wave voltage is achieved by Hall element, when the rotor of motor spindle through Hall element that mounted thereon, Hall element can induce rotor coil, it will produce an electromotive force, the electromotive force has the characteristics of square wave, when the Hall element induce one rotor coil, the current detection generates a square wave voltage ${ }^{[2]}$, the spindle rotates one circle, it will produce eight square wave voltage.

The Hall count mode requires the comparison circuit to obtain, Figure 2 shows the comparator circuit, In order to facilitate the microcontroller or other devices to receive count, the pulse produced by Hall sensors must be converted into pulse that as the benchmark. When the motor voltage collected by Hall sensor is higher than the specified comparison value in the circuit, the system will output 0 low level(the low level is generally lower than $0.7 \mathrm{~V}$ ), on the contrary, the motor voltage collected by Hall sensor is lower than the specified comparison value in the circuit, the system will output 1 high level, so as to realize the output voltage signal is 0,1 in the circuit, and then pass 0,1 voltage signal to the computer, it will complete the counting function.

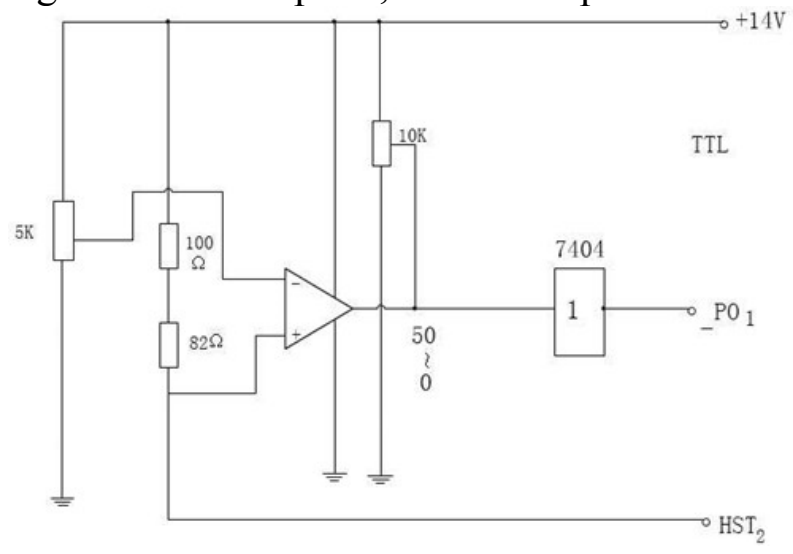

Figure 2 diagram of comparator circuit

In counting with the comparator, once short circuit been under detected components it can burn circuit board easily. Meanwhile, in confirming of detecting parameters, according that whether the number of counting pulse produced by mechanical parts in fixed schedule is within the prescribed scope or not to confirm that memory function of the parts is correct. So all the time of detection, only mechanical components detected go through a full schedule, can it carry out specific judgment. In the factory of today's era, it has a great influence to the scene of the production rhythm. However, the situation that field signal after the AD sampling have square wave detection to realize memory function is carried out by the LabVIEW programming. The system has advantages of developing cycle short, accurate and precise. It is helpful to improve the automation degree of the detection. And it also can completely achieve the judgment of memory function of qualified or not after motor rotation for several cycles.

\section{Digital Filtering Technology And Application}

In the running process of the system, due to the influence of some factors, such as the change of motor internal load, the interference of the external environment and motor parameters or load matching and so on, the data signal of acquisition is the superposition of the useless noise, therefore, after collecting the square wave voltage, this paper use the digital filter for voltage signal to 
eliminate or weaken the noise of the signal.

In general, the design of the filter should start from low pass filter, and then convert them to the desired type of filter through the frequency transformation[3], two kinds of filter characteristics is described below.

Butterworth low-pass filter. Butterworth filter has a smooth pass-band and relatively wide transition zone, it also has good phase-frequency characteristics, it approaches to the ideal low-pass filter with the origin's maximum flatness, the square amplitude function is:

$$
\left|H_{a}(j \Omega)\right|^{2}=\frac{1}{1+\left(W / W_{c}\right)^{2 N}}
$$

In the formula, $N$ is filter orders, $\Omega_{c}$ is cut-off frequency of low-pass filter, When equal $\Omega$ and $\Omega_{\mathrm{c}}$, the module squared of magnitude characteristic $\left|H_{a}(j \Omega)\right|^{2}$ is $1 / 2$, so the $-3 \mathrm{~dB}$ point is filter voltage.

Chebyshev Filter.The magnitude-characteristic of Chebyshev filter is the equiripple, the minimum error between frequency response curve of Chebyshev filter and ideal filter ${ }^{[4]}$, there are two kinds of forms: Chebyshev's model I filter and Chebyshev's model II filter, the square amplitude function of model I is:

$$
\left|H_{\alpha}(j \Omega)\right|^{2}=\frac{1}{1+\varepsilon^{2} C_{N}^{2}\left(\Omega / \Omega_{p}\right)}
$$

In the formula, $\varepsilon$ is less than one positive, indicate the extent of amplitude fluctuation within the pass-band, the greater $\varepsilon$ value, the larger amplitude fluctuation; $\Omega_{\mathrm{p}}$ is pass-band cut-off frequency; $\mathrm{C}_{\mathrm{N}}(\mathrm{x})$ is the second order chebyshev polynomial.

In this paper, considering the real high and low frequency jamming signal, using the Butterworth filter to ensure the reliability and stability of the data.

\section{Square Wave Parameters Detection}

At present, the graphical programming language LabVIEW of NI company uses the icon, attachment and block diagram instead of the traditional program code, observing program flowing visually and intuitively ${ }^{[5]}$. Taking advantage of it to set up all kinds of virtual instrument conveniently. Meanwhile there are beautiful writing-interface, powerful functions, it also realize the data collection, analysis, processing and display of centralized operation. Based on these advantages, it has been widely applied to communications, aeronautics and astronautics, industrial automation and the auto-industry and so on.

Parameter Setting. For square-wave pulse voltage produced by seat motor rotation, what we are concerned about parameters are the square-wave peak, frequency, duty-cycle and duration of the pulse.

Square-wave peak value is the difference of high voltage level value and low voltage level value of signal state at the same time. It is the precondition which must solve in detection and evaluation of signal source, detecting instruments and system's rise time index.

Pulse duration $T_{w}$ is the difference of the time of the crossing between the intermediate reference voltage level and the first two pulse specified. It is also known as pulse width, in seconds $^{[6]}$. Duty-cycle $q$ is the fraction of cycle, also known as pulse duty factor. Essentially it refers to the percentage that the time of controlled circuit connected occupies the entire circuit work cycle. When motor is powered by high input voltage, the speed is faster, the duty ratio is also higher. Its computation formula for: 


$$
q=\frac{T_{W}}{T} \times 100 \%
$$

Cycle $T$ refers to the time interval that the pulse of adjacent two go through the intermediate reference level at same direction, in seconds, its reciprocal is frequency.

The Test Program And Result.The display of square signal by the seat motor's spindle induction and the measurement of signal parameter are implemented in the LabVIEW platform, figure 3 is the square wave pulse voltage of system.

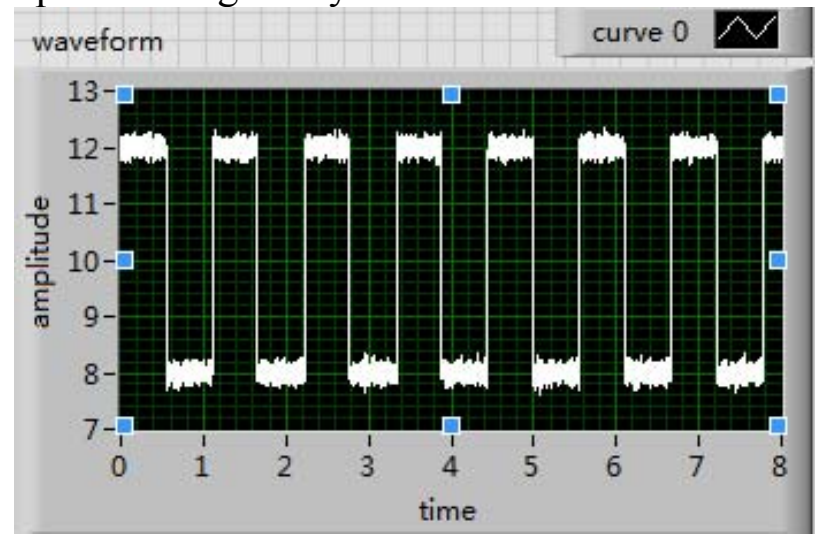

Figure 3 square wave pulse voltage of system

The pulse voltage fluctuates between 8 to $12 \mathrm{~V}$ from above figure, there are a little bit of noise interference. Figure 4 shows the waveform after using digital filter.

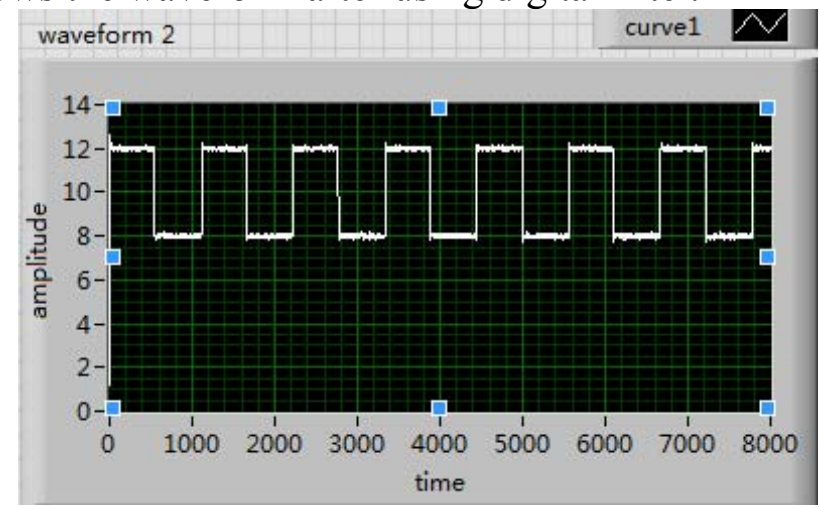

Figure 4 waveform after using digital filter

The design flow chart of test waveform parameters using LabVIEW as shown in Figure 5, press the test button, the program will automatically read the data information that produced by Hall sensor, and then through the filter, calculating the various kinds of parameters at the same time, and compared with the standard values, in the end, testing parameters can meet the requirements to make judgments on motor memory function.

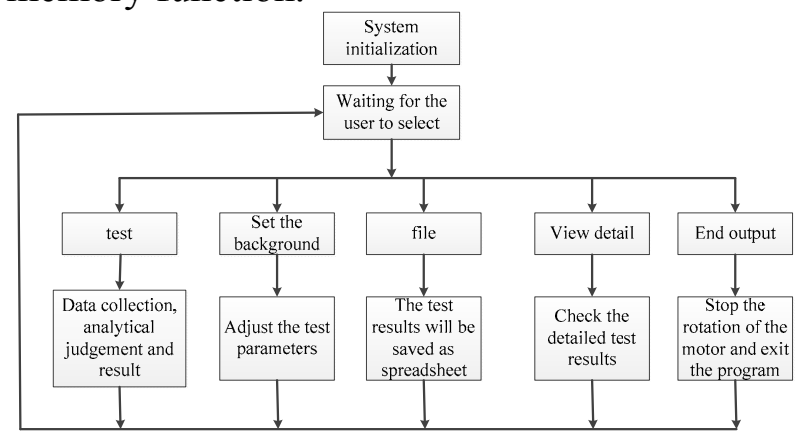

Figure 5 design floe chart of test waveform parameters

In order to ensure to calculate the duty cycle of the square wave, pulse duration and other parameters, the system uses the waveform measurement sub-VI of signal time domain analysis and management provided by LabVIEW in the waveform parameter testing process . 
Connecting the voltage square wave signal to "transient characteristics measurement function controls", the polarity is rising, output is transient duration, in the meantime, the signal is connected to the "pulse function controls", to get the pulse duration and duty-cycle of square wave. While measuring signal frequency, the control can only measure the standard signal, there will be some interference at run time, so use the following formula to calculate the frequency:

$$
f=\frac{n p}{60}
$$

In the formula, $\mathrm{n}$ is motor speed; $\mathrm{p}$ is the number of pulses produced one revolution of the motor. The calculation of the peak will connect signal to the amplitude and level function controls", obtained by subtraction of the high and low level value. The measurement results shows : square wave duty-cycle is about $48 \%$, the pulse duration is 0.53 .

\section{Conclusion}

Constructing the square wave voltage signal from the hall sensor in the motor spindle, the digital filtering technology to process the original square wave signals, using the virtual instrument graphical programming language LabVIEW real-time detecting signals each parameter and judgment, the system has good stability, intuitive and convenient, it can save the test cost and can run for a long time.

\section{Acknowledgements}

This paper is supported by the Jilin science and technology development project (project number: 20130305002GX) and Changchun science and technology project (project number:13KG15).

\section{References}

[1] Wang lei, Pan Hong bing, He Shu zhuan etc, Optimization of integrated hall sensor, J. Electronic measurement technology. 2011 (34)77-78.

[2] Wang $\mathrm{Hu}$, Research on motor current, vibration and memory function text system of cushion, M. Changchun University of Technology, 2012.(3)16-19.

[3] Xing Ji chuan, Wu Jin, Xu Zheng, The principle of digital signal processing and LabVIEW implementation, Electronic industry press,Beijing,2013,pp.194-195.

[4] Wang Fang, Research and application of signal de-noising based on wavelet analysis, M. Xihua University,2009(6)72-74.

[5] Qiu Zheng, Zhang Yong chao, Shen Qi, Design of force sensor data acquisition system based on PXI-4220 and LabVIEW, J. Instrument technique,2013(9)7-8.

[6] Zhou Peng, Xu Gang, Ma Xiao yu etc, Proficient in LabVIEW signal processing, Tsinghua university press, Beijing, 2013, pp.138-139. 